\title{
Continuous Jostling Helps Protein Perform
}

\author{
Kinesin, which moves cargo around inside cells, moves faster with \\ constant buffeting than without, suggesting that it's optimized for the \\ cellular environment.
}

By Philip Ball

$\square$ iological molecules such as enzymes must function in the crowded, bustling environment of the cell, and a new study suggests that they might take advantage of such disturbances to work more effectively [1]. Researchers experimenting with the protein kinesin, which transports molecular cargo along cellular "tracks," found that applying the right kind of shaking force to the molecule caused it to move

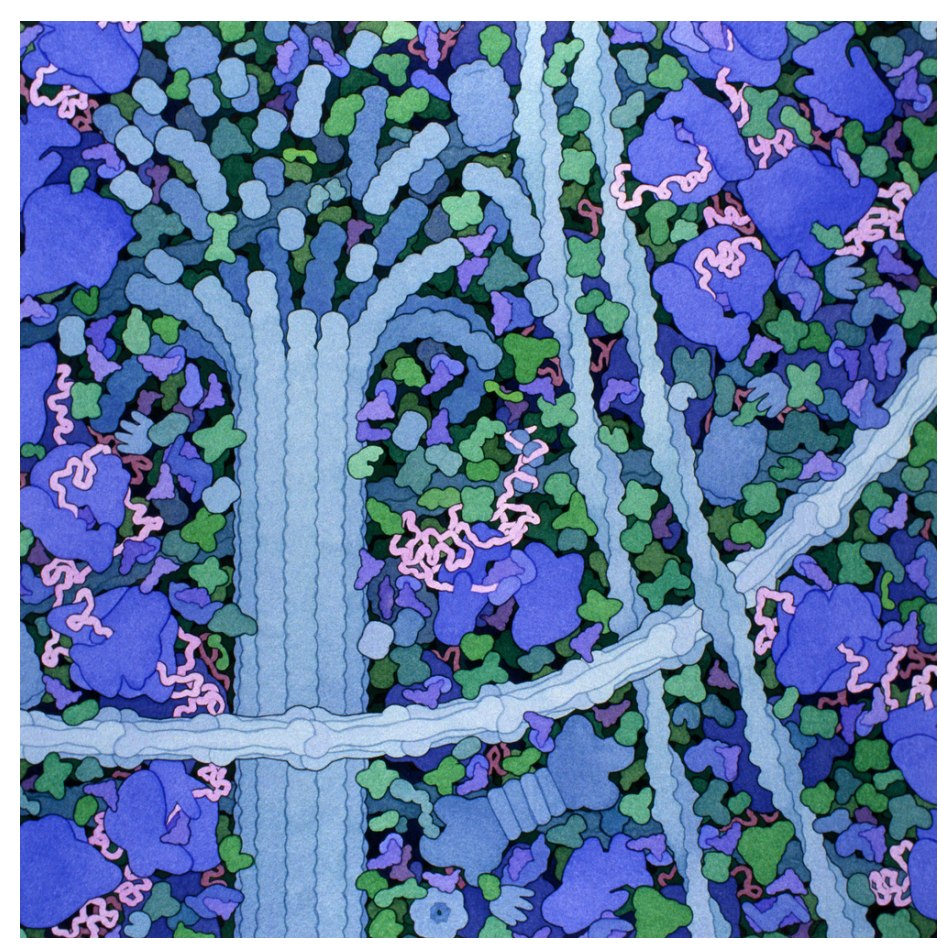

Packed in. This illustration shows the crowded nature of a biological cell. The straight, vertical structure is a microtubule. Credit: D. S. Goodsell (CC BY 4.0) faster. The experiments, along with simulations, suggest that many proteins may perform better in the "noisy" cell interior than in a calm environment outside of cells, where they are typically studied.

Kinesin is a protein that uses energy from ATP molecules to move "packages" called vesicles along rod-like protein assemblies called microtubules. Random thermal fluctuations provide a kind of shaking that helps kinesin move along the microtubules in a specific direction, much as shaking a sieve helps flour grains move through the holes.

Biophysicist Takayuki Ariga of Yamaguchi University in Japan and his co-workers recently reported that kinesin seems surprisingly inefficient. In experiments where isolated kinesin molecules moved along microtubules on a glass surface, they found that about $80 \%$ of the energy consumed from ATP is dissipated as heat, rather than converted into motion [2]. That result seemed odd to the researchers: one would expect such biomolecules to be adapted by evolution to do their job efficiently.

Inside real cells, in addition to thermal noise, molecules are constantly buffeted by active, energy-consuming molecular processes going on around them. In particular, previous experiments have shown that cell interiors are permeated by a type of noise that is different from thermal randomness. It's called Lévy noise, and it consists of constant small random disturbances punctuated every so often by a big jolt. This noise seems to come mainly from another network of filaments and motor proteins, the actomyosin network. 
Walking the walk. This animation illustrates the "walking" motion of a kinesin protein moving along a microtubule.

Credit: G. Johnson/fivth.com/Graham Johnson Medical Media/R. A. Milligan/Scripps Research

To see what effect such noise would have, Ariga and colleagues carried out similar experiments to their earlier ones but with Lévy noise artificially imposed to mimic the cell environment. They attached 500-nanometer-diameter polymer beads, mimicking the vesicle load, to individual kinesin molecules moving on microtubules adsorbed on a glass slide. They then used a focused infrared laser beam to create "optical tweezers," which can trap an individual bead (and the attached kinesin) in an intense light field.

By manipulating the beam's position and brightness, the researchers could cause the force on the kinesin molecule to fluctuate in the manner of Lévy noise. They found that this additional fluctuating force made the protein and its cargo bead move faster, and the speedup increased as the load imposed by the optical tweezers increased.

The team saw the same behavior in computer simulations of kinesin in which they modeled the protein's motion as being driven by the molecule switching between two different conformations. The model suggested that the fluctuating force is able to increase the rates for these switches, regardless of the exact spectrum of those fluctuations.

Acceleration in the presence of noise has been previously seen for the motor protein dynein, which also carries loads along microtubules [3]. However, analyzing the differences in the precise behavior of the two proteins for different applied forces, Ariga and colleagues conclude that the dynein speedup comes from a specific feature of that molecule, whereas the mechanism for kinesin could apply to many other proteins.

In fact, they expect that the same enhancement due to "active" cell noise should be seen in any "protein machine" that undergoes energy-activated changes of conformation, including many enzymes. It is likely to be "a general property of enzymes inside the living cell," says Ariga.
Ahmet Yildiz of the University of California, Berkeley, who conducted the earlier experiments on dynein, says the new research is "high-quality experimental and theoretical work," although he is not yet persuaded that Ariga and colleagues have identified the right mechanism.

Regardless of the mechanism, such enhancement of the efficiency of a dynamical process by noise has been seen for a wide range of physical systems and is called stochastic resonance [4]. Julio Fernández of Columbia University and his co-workers saw it in the protein talin, which senses mechanical forces [5]. "The strikingly similar behavior of these two very different biological systems suggests that molecules operating under force might take advantage of stochastic resonance to enhance their performance in the extremely noisy cellular environment," says Fernández.

Ariga cautions that the relevance of his team's findings for biology remains speculative until further experiments can show that they are also valid inside real cells-which would be very challenging to measure. But Fernández is more confident.

"Most molecules in the body are exposed to mechanical deformation of one sort or another" in noisy settings, he says-and it would make sense for evolution to turn that to advantage. He thinks that if researchers can control such responses to mechanical forces, they may eventually learn to intervene when the relevant biological processes go wrong, for example in some cancers and developmental defects.

Philip Ball is a freelance science writer in London. His latest book is The Modern Myths (University of Chicago Press, 2021).

\section{REFERENCES}

1. T. Ariga et al., "Noise-induced acceleration of single molecule kinesin-1," Phys. Rev. Lett. 127, 178101 (2021).

2. T. Ariga et al., "Nonequilibrium energetics of molecular motor kinesin,” Phys. Rev. Lett. 121, 218101 (2018).

3. Y. Ezber et al., "Dynein harnesses active fluctuations of microtubules for faster movement," Nat. Phys. 16, 312 (2020).

4. L. Gammaitoni et al., "Stochastic resonance," Rev. Mod. Phys. 70, 223 (1998).

5. R. Tapia-Rojo et al., "Talin folding as the tuning fork of cellular mechanotransduction," Proc. Natl. Acad. Sci. U.S.A. 117, 21346 (2020). 